\title{
Características fotossintéticas de Phaseolus vulgaris L.
}

\author{
Carlos Pimentel $^{1,5}$, Ricardo Ferraz de Oliveira ${ }^{2}$, Rafael Vasconcelos Ribeiro ${ }^{3}$, \\ Mauro Guida dos Santos ${ }^{4}$ e Eduardo Caruso Machado ${ }^{3}$
}

Recebido: 5.11.2010; aceito: 16.06 .2011

\begin{abstract}
Photosynthetic characteristics of Phaseolus vulgaris L.). The in vivo analysis of net $\mathrm{CO}_{2}$ assimilation rate (A) versus active photosynthetic photon flux density (DFFFA) and versus intercellular $\mathrm{CO}_{2}$ concentration $\left(\mathrm{C}_{\mathrm{i}}\right)$, allows a more detailed evaluation of the photosynthetic process. The A/DFFFA curve can be used to establish the maximal DFFFA in which the absorbed light energy is quenched in the formation of photochemical products. Using the $\mathrm{A} / \mathrm{C}_{\mathrm{i}}$ curves, it was calculated the maximum rates of Rubisco carboxilation $\left(\mathrm{V}_{\mathrm{c}, \max }\right)$ of $89.3 \mu \mathrm{mol} \mathrm{m}^{-2} \mathrm{~s}^{-1}$, the maximum rates of Rubisco oxygenation $\left(\mathrm{V}_{\mathrm{o}, \max }\right)$ of $24.7 \mu \mathrm{mol} \mathrm{m} \mathrm{m}^{-2}$, the maximum rates of electron transport $\left(\mathrm{J}_{\max }\right)$ of $159.8 \mu \mathrm{mol} \mathrm{m}^{-2} \mathrm{~s}^{-1}$, from which the RuBP regeneration is dependent, the mitochondrial respiration $\left(\mathrm{R}_{\mathrm{d}}\right)$ of $0.88 \mu \mathrm{mol} \mathrm{m}^{-2} \mathrm{~s}^{-1}$, and the mesophyll conductance $\left(\mathrm{g}_{\mathrm{m}}\right)$ of $0.47 \mathrm{~mol} \mathrm{~m}^{-2} \mathrm{~s}^{-1}$ for common bean cultivated in Brazil (Phaseolus vulgaris var. mexicanus).
\end{abstract}

Key words: common bean, mathematical models, Phaseolus vulgaris var. mexicanus

RESUMO - (Características fotossintéticas de Phaseolus vulgaris L.). A análise in vivo da taxa de assimilação líquida de $\mathrm{CO}_{2}(\mathrm{~A})$ em resposta à densidade de fluxo de fótons fotossinteticamente ativos (DFFFA) e em resposta à concentração intercelular de $\mathrm{CO}_{2}$ da folha $\left(\mathrm{C}_{\mathrm{i}}\right)$ permite uma avaliação detalhada do processo fotossintético. Com as curvas A/DFFFA, se estabelece a DFFFA máxima, em que a maior parte da energia absorvida é transferida para a formação de produtos fotoquímicos. Já com as curvas $\mathrm{A} / \mathrm{C}_{\mathrm{i}}$, calculou-se a velocidade máxima de carboxilação da Rubisco $\left(\mathrm{V}_{\mathrm{c} \text {,max }}\right)$ de $89,3 \mu \mathrm{mol} \mathrm{m} \mathrm{m}^{-2} \mathrm{~s}^{-1}$, a velocidade máxima de oxigenação da Rubisco $\left(\mathrm{V}_{\mathrm{o}, \max }\right)$ de $24,7 \mu \mathrm{mol} \mathrm{m} \mathrm{m}^{-2} \mathrm{~s}^{-1}$, a velocidade máxima de transporte de elétrons $\left(\mathrm{J}_{\max }\right)$ de $159,8 \mu \mathrm{mol} \mathrm{m}^{-2} \mathrm{~s}^{-1}$, do qual a regeneração de RuBP é dependente, a respiração mitocondrial $\left(\mathrm{R}_{\mathrm{d}}\right)$ de $0,88 \mu \mathrm{mol} \mathrm{m}^{-2} \mathrm{~s}^{-1}$, e a condutância mesofílica $\left(g_{\mathrm{m}}\right)$ de $0,47 \mathrm{~mol} \mathrm{~m}^{-2} \mathrm{~s}^{-1}$, para o feijoeiro comum cultivado no Brasil (Phaseolus vulgaris var. mexicanus). Palavras-chaves: feijoeiro comum, modelos matemáticos, Phaseolus vulgaris var. mexicanus

\section{Introdução}

A atividade fotossintética de um vegetal com metabolismo $\mathrm{C}_{3}$, como o feijoeiro comum, pode ser avaliada em mais detalhes através das curvas da taxa de assimilação líquida de $\mathrm{CO}_{2}(\mathrm{~A})$, em função da densidade de fluxo de fótons fotossinteticamente ativos (DFFFA) e da concentração intercelular de $\mathrm{CO}_{2}\left(\mathrm{C}_{\mathrm{i}}\right)$. Essa avaliação fica mais completa quando associada com medidas simultâneas de fluorescência da clorofila (Long \& Bernacchi 2003). A curva A/DFFFA ajusta DFFFA máximo a ser usado e a segunda, a curva
$\mathrm{A} / \mathrm{C}_{\mathrm{i}}$ auxilia no cálculo in vivo de variáveis da cinética da Rubisco e da atividade fotoquímica (Bernacchi et al. 2001). Visto que as medidas bioquímicas de atividade da enzima Rubisco são feitas in vitro, estas não se correlacionam com o funcionamento in vivo na planta sob estresses ambientais (Pham Thi et al. 1982). Vassey \& Sharkey (1989) demonstraram uma redução de A de quase $100 \%$ e da condutância estomática $\left(\mathrm{g}_{\mathrm{s}}\right)$ em $60 \%$ no feijoeiro comum sob falta d'água moderada, enquanto a atividade in vitro da Rubisco sob deficiência hídrica severa é pouco alterada (Sage et al. 1988).

1. Universidade Federal Rural do Rio de Janeiro, Instituto de Agronomia, Departamento de Fitotecnia, 23851-970 Seropédica, RJ, Brasil

2. Escola Superior de Agricultura Luiz de Queiroz, Departamento de Ciências Biológicas, Caixa Postal 09, 13419-110 Piracicaba, SP, Brasil

3. Instituto Agronômico, Centro de Pesquisa e Desenvolvimento em Ecofisiologia e Biofísica, Caixa Postal 28, 13001-970 Campinas, SP, Brasil

4. Universidade Federal de Pernambuco, Departamento de Botânica, 50670-901 Recife, PE, Brasil

5. Autor para correspondência: greenman@amcham.com.br 
Apesar dos valores de A obtidos em resposta ao aumento instantâneo de $\mathrm{C}_{\mathrm{i}}$ não serem considerados os mesmos para feijoeiros desenvolvidos sob alta concentração de $\mathrm{CO}_{2}$ do ar, uma única medida instantânea de $\mathrm{A}$ também não fornece muitas informações sobre a atividade do aparato fotossintético, em suas etapas fotoquímica e bioquímica e, por conseqüência, sobre o estado fisiológico da planta (Pimentel et al. 2007). Contudo os modelos matemáticos apresentados por Bernacchi et al. (2001) permitem uma análise mais completa das etapas fotoquímica e bioquímica da fotossíntese, pois os modelos antigos (Farquhar et al. 1980), usados nos cálculos de $\mathrm{A}$, assumem que $\mathrm{C}_{\mathrm{c}}$ é igual à $\mathrm{C}_{\mathrm{i}}$, o que não é exato, pois há a respiração mitocondrial e a fotorrespiração, que devem ser levadas em conta no cálculo de $\mathrm{C}_{\mathrm{c}}$, além de não poderem ser aplicados a temperaturas ambiente diferentes de $25^{\circ} \mathrm{C}$ (Long \& Bernacchi 2003). Além disso, algumas variáveis fotoquímicas, calculadas a partir das medidas da emissão de fluorescência da clorofila, são mais sensíveis às variações ambientais instantâneas que as trocas gasosas (Pimentel et al. 2004). Isto torna a estimativa das variáveis bioquímicas calculadas pelas curvas $\mathrm{A} / \mathrm{C}_{\mathrm{i}}$ mais robusta e confiável, quando associadas às medidas de fluorescência, acrescentado mais informações sobre a fotossíntese que apenas as medidas de trocas gasosas (Long \& Bernacchi 2003).

Por outro lado, a maioria dos experimentos na literatura, para fotossíntese em feijão comum, foram realizados nos Estados Unidos ou na Europa, onde se cultiva, principalmente, genótipos de Phaseolus vulgaris var. aborigineus, com centro de origem no sul dos Andes, grãos grandes, proteína phaseolina tipo "S", e adaptados às temperaturas mais baixas; enquanto no Brasil se cultiva genótipos de Phaseolus vulgaris var. mexicanus, com centro de origem no México, grãos pequenos, proteína phaseolina tipo " $\mathrm{T}$ ", e adaptados às temperaturas mais elevadas, segundo Norman et al. (1995). Assim sendo, o objetivo do presente estudo foi caracterizar in vivo a atividade fotossintética do genótipo de feijoeiro comum mais cultivado no Brasil, o Carioca, considerando as variáveis fisiológicas obtidas pelas medidas simultâneas da resposta de $\mathrm{A}$ e da atividade fotoquímica ao aumento de DFFFA e de $\mathrm{C}_{\mathrm{i}}$; e comparar estes resultados obtidos com Phaseolus vulgaris var. mexicanus, aos da maioria da literatura, com Phaseolus vulgaris var. aborigineus.

\section{Material e métodos}

O genótipo de Phaseolus vulgaris raça mexicanus escolhido para a caracterização foi a cv Carioca, mais cultivada e consumida no Brasil (Vieira 1998). As sementes desta cultivar foram semeadas em potes de $8 \mathrm{~L}$ (uma planta por pote), em substrato composto por matéria orgânica misturada com vermiculita expandida (Plantimax - Eucatex Inc., Brasil), na proporção de $3: 1$. As plantas foram crescidas em casa de vegetação, com temperatura variando entre 12 (mínima noturna) e 28 (máxima diurna) ${ }^{\circ} \mathrm{C}$. Antes do plantio, os substratos foram adubados (doses equivalentes) com N (20 kg ha'), $\mathrm{P}_{2} \mathrm{O}_{5}\left(90 \mathrm{~kg} \mathrm{ha}^{-1}\right), \mathrm{K}_{2} \mathrm{O}\left(30 \mathrm{~kg} \mathrm{ha}^{-1}\right)$ e calcário dolomítico (1,5 $\left.\mathrm{Mg} \mathrm{ha}^{-1}\right)$; e com micronutrientes (300 mL de solução de Hoagland), como proposto por McCree (1986). Uma adubação nitrogenada de cobertura foi realizada aos 25 dias após a emergência (DAE), usando uma dose equivalente a $40 \mathrm{~kg} \mathrm{~N} \mathrm{ha}^{-1}$ de uréia solúvel. Estas doses de fertilizantes são usuais na cultura do feijoeiro comum (Vieira 1998).

As medidas de fotossíntese foram feitas na fase de pré-floração das plantas (40 a 45 dias após o plantio). As avaliações in vivo da cinética enzimática da Rubisco, da assimilação fotossintética do $\mathrm{CO}_{2} \mathrm{e}$ da atividade fotoquímica foram feitas conforme modelos matemáticos propostos por Farquhar et al. (1980), ajustados por Bernacchi et al. (2001) e descritas em Long \& Bernacchi (2003), baseadas em medidas simultâneas de trocas gasosas e de fluorescência da clorofila: velocidade máxima de carboxilação $\left(\mathrm{V}_{\mathrm{c}, \max }\right)$ e de oxigenação $\left(\mathrm{V}_{\mathrm{o} \text {,max }}\right)$ da Rubisco, velocidade máxima de transporte de elétrons $\left(\mathrm{J}_{\max }\right)$, taxa de assimilação líquida de $\mathrm{CO}_{2}$ máxima $\left(\mathrm{A}_{\max }\right)$, a $380 \mu \mathrm{mol} \mathrm{mol}^{-1}$ de $\mathrm{CO}_{2}$, respiração mitocondrial $\left(\mathrm{R}_{\mathrm{d}}\right)$ e condutância mesofílica $\left(\mathrm{g}_{\mathrm{m}}\right)$. Curvas de resposta da taxa de assimilação líquida de $\mathrm{CO}_{2}$ (A) ao aumento da densidade de fluxo de fótons fotossinteticamente ativos (DFFFA) e da concentração intercelular de $\mathrm{CO}_{2}\left(\mathrm{C}_{\mathrm{i}}\right)$ foram realizadas variando-se DFFFA e a concentração de $\mathrm{CO}_{2}$ do ar na câmara de medida. Medidas simultâneas de atividade fotoquímica também foram realizadas com um fluorômetro, calculando as variáveis derivadas do padrão de emissão de fluorescência descritas por Maxwell \& Johnson (2000) e Rohacek (2002): eficiência quântica potencial $\left(\mathrm{F}_{\mathrm{v}} / \mathrm{F}_{\mathrm{m}}\right)$ e efetiva $\left(\Phi_{\mathrm{PSII}}\right)$ do fotossistema II, o transporte aparente de elétrons (ETR) e os coeficientes de extinção fotoquímica (qP) 
e não fotoquímica (qN e NPQ) da fluorescência. Enquanto qN é mais sensível ao estado de energização da membrana dos tilacóides, NPQ é mais apropriado para a avaliação da dissipação da energia de excitação, não fotoquímica, na forma de calor no sistema antena do FSII (Schreiber et al. 1994).

As medidas de A, a condutância estomática $\left(g_{s}\right)$ e a fluorescência da clorofila foram feitas com um analisador de gases por infravermelho, com fluorômetro acoplado à câmara de medidas (LI-6400XT, Li-Cor, EUA), em sistema aberto, com uma câmara de $6 \mathrm{~cm}^{2}$. As medidas foram realizadas entre 10:00 e 11:30 h, no folíolo central da quinta folha trifoliolada, que era a mais jovem com expansão foliar máxima.

Após uma hora de aclimatação a DFFFA de $1.500 \mu \mathrm{mol} \mathrm{m} \mathrm{m}^{-2} \mathrm{~s}^{-1}$, a curva A/DFFFA foi iniciada e DFFFA diminuído gradativamente (em intervalos de $50 \mu \mathrm{mol} \mathrm{m} \mathrm{m}^{-2} \mathrm{~s}^{-1}$ ), aguardando 5 min em cada DFFFA. A variação de DFFFA foi controlada com uma fonte de luz LED vermelho-azul acoplada sobre a câmara. Nas curvas $A / C_{i}$, DFFFA foi mantido constante a $700 \mu \mathrm{mol} \mathrm{m} \mathrm{s}^{-2}$, como determinado previamente na curva A/DFFFA, e a concentração de $\mathrm{CO}_{2}$ foi modificada gradualmente. A concentração inicial de $\mathrm{CO}_{2}$ no ar na câmara $(\mathrm{Ca})$ foi ajustada para $380 \mu \mathrm{mol} \mathrm{mol}^{-1}$, sendo reduzida gradualmente até $50 \mu \mathrm{mol} \mathrm{mol}^{-1}$, para voltar em seguida a $380 \mu \mathrm{mol} \mathrm{mol}^{-1}$ e aumentar gradualmente até $1.500 \mu \mathrm{mol} \mathrm{mol}^{-1}$. Este procedimento é realizado para evitar oscilações estomáticas ("patchness"), principalmente sob altos valores de $\mathrm{C}_{\mathrm{a}}$ (Pimentel et al. 2007). A medida de

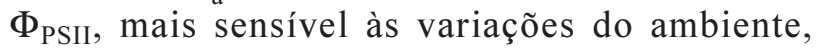
simultânea à de A foi usada para confirmar a estabilidade temporal das medidas de trocas gasosas. $\mathrm{O}$ ar direcionado para a câmara de medida foi coletado fora da casa de vegetação, tendo aproximadamente

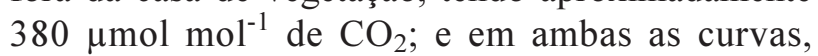
$\mathrm{A} / \mathrm{DFFFA} \mathrm{e} \mathrm{A} / \mathrm{C}_{\mathrm{i}}$, o déficit de pressão de vapor do ar (DPV) dentro da câmara foi mantido inferior a 2,0 kPa, para prevenir o fechamento estomático, assim como a temperatura da câmara foi mantida a $25 \pm 1{ }^{\circ} \mathrm{C}$, conforme descrito em Pimentel et al. (2004, 2007).

$\mathrm{O}$ experimento foi conduzido em delineamento experimental completamente casualizado, com quatro repetições das curvas A/DFFFA e $\mathrm{A} / \mathrm{C}_{\mathrm{i}}$ feitas em plantas distintas. Para a análise estatística foi realizada uma análise de variância e a comparação entre as médias foi feita pelo teste de Student-Newman-Keuls, a $5 \%$ de probabilidade, obtendo-se o desvio padrão das médias e verificando-se se houveram efeitos significativos, nas variáveis fotossintéticas, causados pelo aumento de DFFFA ou $\mathrm{C}_{\mathrm{i}}$.

\section{Resultados e Discussão}

A análise in vivo da fotossíntese do feijoeiro comum, através das curvas A/DFFFA e $A / C_{i}$, calculando todas as variáveis propostas por Long $\&$ Bernacchi (2003), inclusive a $g_{m}$, foi feita, no presente trabalho, no genótipo mais cultivado no Brasil, o Carioca, de Phaseolus vulgaris var. mexicanus; enquanto a maioria dos resultados apresentados na literatura foi obtida em genótipos de Phaseolus vulgaris var. aborigineus, segundo a descrição de Norman et al. (1995). Antes de proceder à análise da fotossíntese, a medida de $\mathrm{F}_{\mathrm{v}} / \mathrm{F}_{\mathrm{m}}$ permitiu a certificação de que a folha escolhida estava em bom estado fisiológico, i.e., com valores entre 0,75 e 0,85 (Schreiber et al. 1994). No presente estudo, o genótipo Carioca apresentou $\mathrm{F}_{\mathrm{v}} / \mathrm{F}_{\mathrm{m}}$ de $0,78 \pm 0,02$, semelhante aos valores apresentados por Santos et al. (2009) e por Wentworth et al. (2006), em plantas não estressadas.

Em seguida, fizeram-se as medidas de A em função de DFFFA, acompanhadas de medidas da ETR (figura 1) e de outras variáveis fotoquímicas (figuras $2,3)$. A ETR é uma medida da atividade fotoquímica real dos fotossistemas e não da atividade potencial máxima, como $\mathrm{F}_{\mathrm{v}} / \mathrm{F}_{\mathrm{m}}$ (Santos et al. 2009). Essa última variável é menos sensível às variações instantâneas das condições ambientais se comparada a ETR ou a $\Phi_{\text {PSII }}$, mas é indicadora de efeitos de estresses graduais abióticos e bióticos (Maxwell \& Johnson 2000, Rohacek 2002). Os valores máximos de A e ETR sob saturação luminosa encontrados para Carioca foram de $20 \mu \mathrm{mol} \mathrm{m}{ }^{-2} \mathrm{~s}^{-1}$ e de $120 \mu \mathrm{mol} \mathrm{m}^{-2} \mathrm{~s}^{-1}$, respectivamente, sem variação significativa para DFFFA acima de $800 \mu \mathrm{mol} \mathrm{m}^{-2} \mathrm{~s}^{-1}$ (figura 1). Os valores de A máxima a $380 \mu \mathrm{mol} \mathrm{mol}^{-1}$ de $\mathrm{CO}_{2}$ no $\operatorname{ar}\left(\mathrm{A}_{\max }\right.$, sob concentração atual de $\mathrm{CO}_{2}$ ) desse genótipo, de Phaseolus vulgaris var. mexicanus, são semelhantes aos apresentados por O'Toole et al. (1977), Lynch et al. (1992) e Sage et al. (1988), assim como a resposta de A ao aumento de DFFFA foi comparável àquelas apresentadas por Sage et al. (1988) e Wentworth et al. (2006), mas para Phaseolus vulgaris, var. aborigineus, não havendo diferenças para A entre os dois grupos de Phaseolus vulgaris, de origem e base genética distintas (Norman et al. 1995). Contudo, nesses dois 


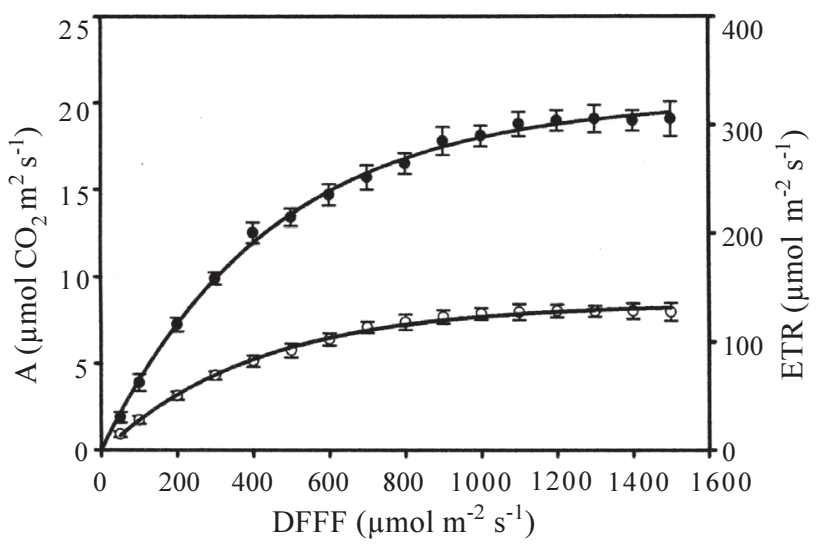

Figura 1. Curvas de resposta da taxa de assimilação líquida do $\mathrm{CO}_{2}$ (A) ao aumento na densidade de fluxo de fótons fotossinteticamente ativos (DFFFA), usadas para estabelecer a saturação luminosa de $\mathrm{A}(\bullet)$, e a velocidade de transporte de elétrons (ETR)/DFFFA (O), usadas para aferir as medidas de A/DFFFA, em feijoeiro comum a $25^{\circ} \mathrm{C}$ e $380 \mu \mathrm{mol} \mathrm{mol}^{-1}$ de $\mathrm{CO}_{2}$ (médias \pm desvio padrão, $\mathrm{n}=4$ ).

Figure 1. Curves of the response of the photosynthetic $\mathrm{CO}_{2}$ assimilation rate (A) to increasing active photosynthetic photon flux density (DFFFA), to establish light saturation of A $(\bullet)$, and the electron transport rate (ETR) per DFFFA (O), to be used for ensuring the measurements of A/DFFFA, in common bean at $25^{\circ} \mathrm{C}$ and $380 \mu \mathrm{mol} \mathrm{mol}^{-1}$ of $\mathrm{CO}_{2}$ (means \pm standard deviation, $\mathrm{n}=4$ ).

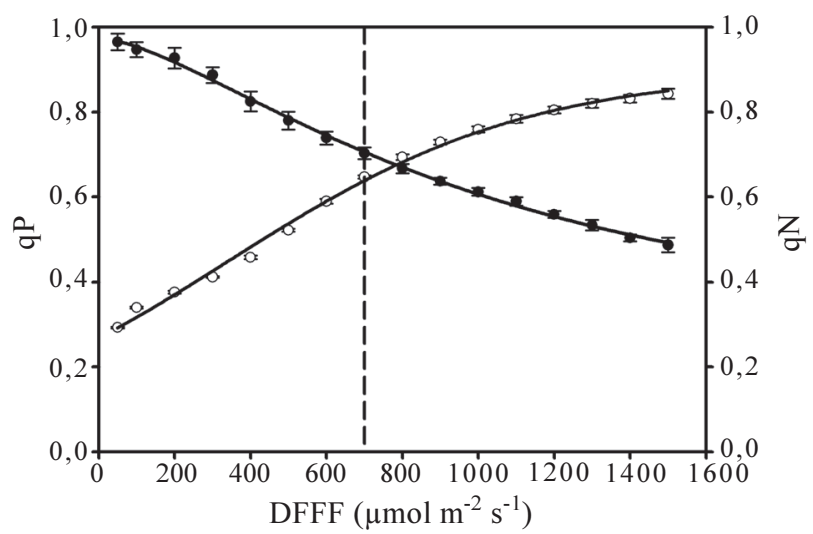

Figura 2. Curvas de resposta do coeficiente de extinção fotoquímica $(\mathrm{qP},[\bullet])$, e do coeficiente de extinção não fotoquímica (qN [o]) ao aumento na densidade de fluxo de fótons fotossinteticamente ativos (DFFFA), obtidas por medidas de fluorescência, em feijoeiro comum a $25^{\circ} \mathrm{C}$ e $380 \mu \mathrm{mol} \mathrm{mol}^{-1}$ de $\mathrm{CO}_{2}(\mathrm{n}=4)$.

Figure 2. Curves of the response of photochemical quenching $(\mathrm{qP},[\bullet])$, and non-photochemical quenching $(\mathrm{qN}[\mathrm{o}])$ coefficients to increasing active photosynthetic photon flux density (DFFFA), obtained by fluorescence measurements, in common bean at $25^{\circ} \mathrm{C}$ and $380 \mu \mathrm{mol} \mathrm{mol}^{-1}$ of $\mathrm{CO}_{2}(\mathrm{n}=4)$.

últimos trabalhos citados com Phaseolus vulgaris var. aborigineus, o valor de $\mathrm{A}_{\max }$ obtido foi de $15 \mu \mathrm{mol} \mathrm{m} \mathrm{s}^{-2} \mathrm{~s}^{-1}$ e os máximos de $\mathrm{g}_{\mathrm{s}}$, encontrados por Wentworth et al. (2006), foram de $0,32 \mathrm{~mol} \mathrm{~m}^{-2} \mathrm{~s}^{-1}$. Além disso, esses autores também realizaram curvas

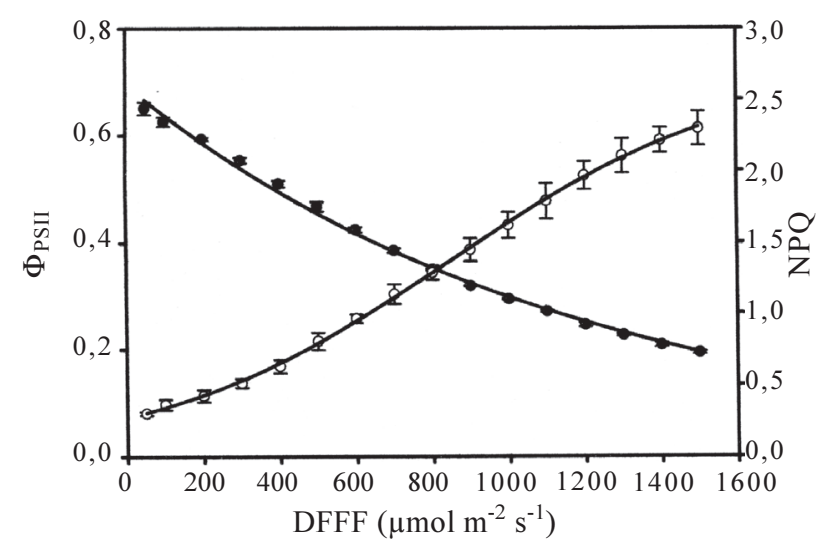

Figura 3. Curvas de resposta do rendimento quântico efetivo $\left(\Phi_{\mathrm{PSII}} \bullet^{\bullet}\right)$ e da dissipação não fotoquímica (NPQ: ○) ao aumento na densidade de fluxo de fótons fotossinteticamente ativos (DFFFA), obtido por medidas de fluorescência, em feijoeiro

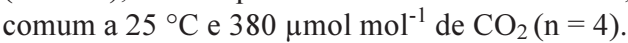

Figure 3. Curves of the response of the efficiency of PS II photochemistry $\left(\Phi_{\mathrm{PSII}}: \bullet\right)$ and the non-photochemical quenching (NPQ: $\odot$ ) to increasing active photosynthetic photon flux density (DFFFA), obtained by fluorescence measurements, in common bean at $25^{\circ} \mathrm{C}$ and $380 \mu \mathrm{mol} \mathrm{mol}{ }^{-1}$ of $\mathrm{CO}_{2}(\mathrm{n}=4)$.

A/DFFFA e A/C $C_{i}$, Sage et al. (1988) calcularam $V_{c, \max }$ e $J_{\max }$, mas não calcularam as outras variáveis da fotossíntese in vivo apresentadas na tabela 1. Já Santos et al. (2009), com Phaseolus vulgaris var. mexicanus, encontraram valores mais baixos de ETR, em torno de $70 \mu \mathrm{mol} \mathrm{m}{ }^{-2} \mathrm{~s}^{-1}$, também em plantas com $\mathrm{A}_{\max }$ em torno de $15 \mu \mathrm{mol} \mathrm{m}^{-2} \mathrm{~s}^{-1}$ mas com $\mathrm{g}_{\mathrm{s}}$ máxima de $0,4 \mathrm{~mol} \mathrm{~m}^{-2} \mathrm{~s}^{-1}$. Portanto, valores de $\mathrm{A}_{\max }$ entre 10 e $25 \mu \mathrm{mol} \mathrm{m}^{-2} \mathrm{~s}^{-1}$ e de $\mathrm{g}_{\mathrm{s}}$ entre 0,1 e $0,4 \mathrm{~mol} \mathrm{~m}^{-2} \mathrm{~s}^{-1}$ são comuns nos dois grupos de Phaseolus vulgaris var. mexicanus e var. aborigineus, dependendo das condições de cultivo (O'Toole et al. 1977, Sage et al. 1988, Vassey \& Sharkey 1989, Pimentel et al. 1999).

Nas curvas A/DFFFA (figura 1) foi possível identificar a DFFFA saturante de A, a partir da qual não houve mais diferença significativa para $\mathrm{A}$, com balanço adequado entre os processos fotoquímicos e não fotoquímicos de dissipação de energia, i.e., em que qP é maior que a qN (figura 2). No presente estudo com Carioca, com DFFFA acima de $700 \mu \mathrm{mol} \mathrm{m}^{-2} \mathrm{~s}^{-1}$, qN foi igual ou maior que qP. Essa variável qP está associada ao número de centros de reação do fotossistema II (FSII) abertos, número que diminui em função da saturação luminosa, enquanto $\mathrm{qN}$ reflete a ativação de processos não fotoquímicos de dissipação de energia por calor (Maxwell \& Johnson 2000). A medida de qP, junto com a de qN, que tem valores na mesma escala (Rohacek 2002), serviu para discriminar respostas de genótipos de feijoeiro comum 
Tabela 1. Variáveis para caracterização in vivo da cinética enzimática da Ribulose- 1,5- Bisfosfato Carboxilase-Oxigenase (Rubisco), da fotossíntese máxima e da atividade fotoquímica: velocidade máxima de carboxilação $\left(\mathrm{V}_{\mathrm{c}, \max }\right)$ e de oxigenação $\left(\mathrm{V}_{\mathrm{o}, \max }\right)$ da $\mathrm{Rubisco}$, velocidade máxima de transporte de elétrons $\left(\mathrm{J}_{\max }\right)$, assimilação de $\mathrm{CO}_{2}$ líquida máxima $\left(\mathrm{A}_{\max }\right)$, a $380 \mu$ mol mol ${ }^{-1}$ de $\mathrm{CO}_{2}$, respiração mitocondrial $\left(\mathrm{R}_{\mathrm{d}}\right)$ e condutância mesofílica $\left(\mathrm{g}_{\mathrm{m}}\right)$, determinadas por medidas de trocas gasosas, na cultivar Carioca de feijoeiro comum, sem estresses $\left(\mathrm{F}_{\mathrm{v}} / \mathrm{F}_{\mathrm{m}}=0,78 \pm 0,02\right)$, a $25^{\circ} \mathrm{C}$.

Table 1. Parameters for in vivo characterization of the enzymatic kinetic of Ribulose- 1,5- Bisphosphate Carboxylase-Oxygenase (Rubisco), the maximal photosynthesis and photochemical activity: maximal Rubisco carboxylation rate $\left(\mathrm{V}_{\mathrm{c} \text {, max }}\right)$ and oxygenation rate $\left(\mathrm{V}_{\mathrm{o}, \max }\right)$, maximal electron transport rate $\left(\mathrm{J}_{\max }\right)$, maximal photosynthetic $\mathrm{CO}_{2}$ assimilation rate $\left(\mathrm{A}_{\max }\right)$, at $380 \mu \mathrm{mol}$ mol ${ }^{-1}$ of $\mathrm{CO}_{2}$, mitochondrial respiration $\left(\mathrm{R}_{\mathrm{d}}\right)$ and mesophylic conductance $\left(\mathrm{g}_{\mathrm{m}}\right)$, obtained by gas exchange measurements, in common bean cultivar Carioca, without stresses $\left(\mathrm{F}_{\mathrm{v}} / \mathrm{F}_{\mathrm{m}}=0,78 \pm 0,02\right)$, at $25^{\circ} \mathrm{C}$.

\begin{tabular}{|c|c|c|c|c|c|}
\hline 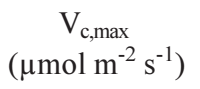 & 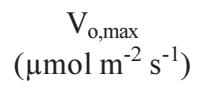 & $\begin{array}{c}\mathrm{J}_{\max } \\
\left(\mu \mathrm{mol} \mathrm{m} \mathrm{m}^{-2} \mathrm{~s}^{-1}\right)\end{array}$ & $\begin{array}{c}\mathrm{A}_{\max } \\
\left(\mu \mathrm{mol} \mathrm{m} \mathrm{m}^{-2} \mathrm{~s}^{-1}\right)\end{array}$ & $\begin{array}{c}\mathrm{R}_{\mathrm{d}} \\
\left(\mu \mathrm{mol} \mathrm{m} \mathrm{m}^{-2} \mathrm{~s}^{-1}\right)\end{array}$ & $\frac{\mathrm{g}_{\mathrm{m}}}{\left(\mathrm{mol} \mathrm{m} \mathrm{m}^{-2} \mathrm{~s}^{-1}\right)}$ \\
\hline $89,3 \pm 3,4$ & $24,7 \pm 1,5$ & $159,9 \pm 2,9$ & $20,5 \pm 2,2$ & $0,9 \pm 0,1$ & $0,47 \pm 0,1$ \\
\hline
\end{tabular}

sob estresse térmico ou por seca (Pastenes \& Horton 1996, Santos et al. 2009).

Com o aumento da DFFFA, houve redução da eficiência quântica efetiva do fotossistema II ( $\left.\Phi_{\mathrm{PSII}}\right)$ e aumento do coeficiente de extinção não-fotoquímica da fluorescência (NPQ) (figura 3), com valores superiores e mais sensível que o qN (Maxwell \& Johnson, 2000), com redução de 50\% do valor inicial da $\Phi_{\text {PSII }}$ e aumento proporcional de NPQ em DFFFA acima de $700 \mu \mathrm{mol} \mathrm{m} \mathrm{m}^{-2} \mathrm{~s}^{-1}$. A NPQ representa a acidificação do lúmem dos tilacóides associado com o ciclo das xantofilas, um mecanismo de fotoproteção (Maxwell \& Johnson 2000). Já o $\Phi_{\text {PSII }}$, uma estimativa do rendimento quântico fotoquímico do PSII relacionado ao transporte de elétrons não cíclico, e o ETR são as variáveis melhor indicadoras da atividade fotoquímica real (Genty et al. 1989). Após determinação de DFFFA máxima com a maior parte da energia sendo direcionada para eventos fotoquímicos primários, realizou-se medidas de $\mathrm{A}$ em função de $C_{i}$ associadas com a medida de $\Phi_{\text {PSII }}$ (figura 4), indicadora de efeitos de oscilações estomáticas e estresses ambientais na câmara de medidas (Long \& Bernacchi 2003). A variação proporcional de A e de $\Phi_{\text {PSII }}$ ou ETR, em função de DFFFA (figura 1) ou $C_{i}$ (figura 4), serviu como indicador de que não houve oscilações de $\mathrm{g}_{\mathrm{s}}$, por exemplo, o que afetaria mais rapidamente os valores de $\Phi_{\text {PSII }}$ ou ETR que os de A, como apresentado por Pimentel et al. (2004).

$\mathrm{Na}$ cultivar Carioca de feijoeiro comum, A saturou-se a $25 \mu \mathrm{mol} \mathrm{m} \mathrm{m}^{-2} \mathrm{~s}^{-1}$ a partir de uma $\mathrm{C}_{\mathrm{i}}$ de $600 \mu \mathrm{mol} \mathrm{mol}^{-1}$ (figura 4), com $\mathrm{g}_{\mathrm{s}}$ máxima de $0,4 \mathrm{~mol} \mathrm{~m}^{-2} \mathrm{~s}^{-1}$. A saturação de A ocorre quando a $\mathrm{C}_{\mathrm{a}}$ elevada elimina a fotorrespiração e a regeneração do substrato RuBP, para a carboxilação da Rubisco, passa a ser limitada pela atividade fotoquímica (Sage et al. 1988, Long \& Bernacchi 2003). Com as curvas $\mathrm{A} / \mathrm{C}_{\mathrm{i}}$ da cultivar Carioca, obteve-se valores de velocidade máxima de carboxilação da Rubisco $\left(\mathrm{V}_{\mathrm{c}, \text { max }}\right)$ de $89,3 \mu \mathrm{mol} \mathrm{m} \mathrm{m}^{-2} \mathrm{~s}^{-1}$, de transporte de elétrons $\left(\mathrm{J}_{\max }\right)$ de $159,8 \mu \mathrm{mol} \mathrm{m} \mathrm{m}^{-2} \mathrm{~s}^{-1}$ e de respiração mitocondrial $\left(\mathrm{R}_{\mathrm{d}}\right)$ de $0,88 \mu \mathrm{mol} \mathrm{m} \mathrm{m}^{-2} \mathrm{~s}^{-1}$ (tabela 1 ). Estes valores obtidos no presente estudo, com Phaseolus vulgaris var. mexicanus, são semelhantes aqueles obtidos com Phaseolus vulgaris var. aborigineus, apresentados por

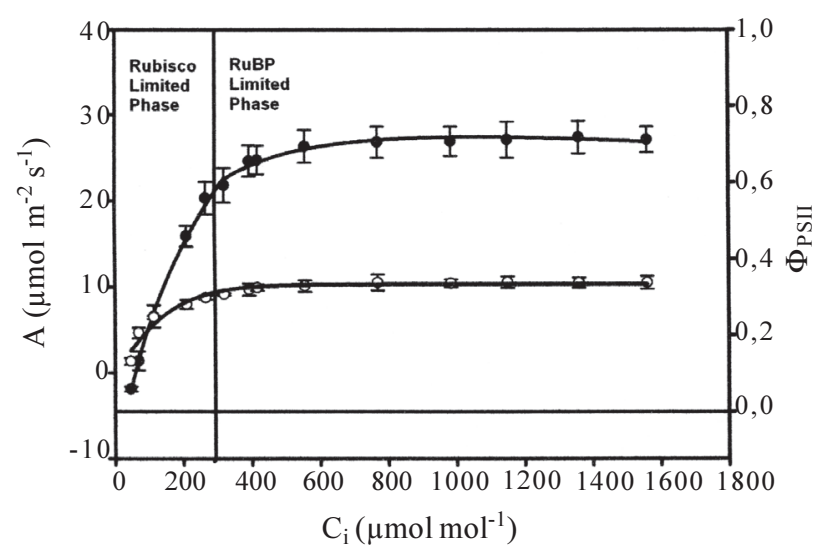

Figura 4. Curvas de resposta da taxa de assimilação líquida do $\mathrm{CO}_{2}(\mathrm{~A}[\bullet])$ e do rendimento quântico efetivo $\left(\Phi_{\mathrm{PSII}}[\mathrm{o}]\right)$ ao aumento da concentração intercelular do $\mathrm{CO}_{2}\left(\mathrm{C}_{\mathrm{i}}\right)$, usadas para estabelecer as curvas $\mathrm{A} / \mathrm{C}_{\mathrm{i}}$, usada para obter-se as variáveis da tabela 1 , e as curvas $\Phi_{\text {PSII }} / \mathrm{C}_{\mathrm{i}}$, para aferir as medidas de $\mathrm{A} / \mathrm{C}_{\mathrm{i}}$, em feijoeiro comum, a $25^{\circ} \mathrm{C}$ e DFFFA de $700 \mu \mathrm{mol} \mathrm{m}{ }^{-2} \mathrm{~s}^{-1}(\mathrm{n}=4)$.

Figure 4. Curves of the response of the photosynthetic $\mathrm{CO}_{2}$ assimilation rate $(\mathrm{A}[\bullet])$ and the efficiency of PS II photochemistry $\left(\Phi_{\mathrm{PSII}}[\circ]\right)$ to increasing $\mathrm{CO}_{2}$ intercellular concentration $\left(\mathrm{C}_{\mathrm{i}}\right)$ to establish the $\mathrm{A} / \mathrm{C}_{\mathrm{i}}$ curves, used to obtain the Table 1 parameters, and the $\Phi_{\text {PSII }} / C_{i}$ curves to ensure the $\mathrm{A} / \mathrm{C}_{\mathrm{i}}$ curves, in common bean a $25^{\circ} \mathrm{C}$ and DFFFA of $700 \mu \mathrm{mol} \mathrm{m}^{-2} \mathrm{~s}^{-1}$ of $\mathrm{CO}_{2}(\mathrm{n}=4)$. 
Wullschleger (1993), O'Toole et al. (1977), Sage et al. (1988) e Lynch et al. (1992). Os valores de $\mathrm{V}_{\mathrm{c} \text {, max }}$ da Rubisco e de $\mathrm{J}_{\max }$ obtidos no presente estudo, estão acima da média de 64 e $134 \mu \mathrm{mol} \mathrm{m}^{-2} \mathrm{~s}^{-1}$, respectivamente, de 109 espécies de plantas $C_{3}$, porém, estes valores estão entre 49 e $91 \mu \mathrm{mol} \mathrm{m}^{-2} \mathrm{~s}^{-1}$ e 96 e $165 \mu \mathrm{mol} \mathrm{m} \mathrm{m}^{-2} \mathrm{~s}^{-1}$, respectivamente, como apresentado por Wullschleger (1993) para o feijoeiro comum.

A variável fotossintética menos estudada pela análise in vivo da fotossíntese é a $\mathrm{g}_{\mathrm{m}}$, pois até recentemente não havia métodos mais simples para calculá-la (Long \& Bernacchi 2003). Essa variável fotossintética é importante para se avaliar a concentração de $\mathrm{CO}_{2}$ no cloroplasto $\left(\mathrm{C}_{\mathrm{c}}\right)$, onde está a Rubisco, permitindo uma estimativa mais real da atividade da Rubisco (Long \& Bernacchi 2003). No atual estudo, a cultivar Carioca apresentou $\mathrm{g}_{\mathrm{m}} \mathrm{de}$ $0,47 \mathrm{~mol} \mathrm{~m}^{-2} \mathrm{~s}^{-1}$, o que está na média dos valores de $\mathrm{g}_{\mathrm{m}}$ para plantas herbáceas, entre 0,16 e $0,39 \mathrm{~mol} \mathrm{~m}^{-2} \mathrm{~s}^{-1}$ (Flexas et al. 2008). Esses valores foram provavelmente obtidos com $\mathrm{g}_{\mathrm{s}}$ baixa, menor que $0,3 \mathrm{~mol} \mathrm{~m}^{-2} \mathrm{~s}^{-1}$ (Wentworth et al. 2006), enquanto que no presente estudo, os valores de $\mathrm{g}_{\mathrm{s}}$ foram maiores que $0,4 \mathrm{~mol} \mathrm{~m}^{-2} \mathrm{~s}^{-1}$. Assim, foi caracterizada a atividade fotossintética in vivo máxima para o feijoeiro comum, cultivar Carioca, a $25{ }^{\circ} \mathrm{C}$, em que $A_{\max }$ foi de $20 \mu \mathrm{mol} \mathrm{m} \mathrm{m}^{-2} \mathrm{~s}^{-1}$, sendo que A saturou-se a $25 \mu \mathrm{mol} \mathrm{m} \mathrm{s}^{-2}$, acima de 600 a $800 \mu \mathrm{mol} \mathrm{mol}^{-1} \mathrm{de} \mathrm{CO}_{2}$ no ar, a $\mathrm{g}_{\mathrm{s}}$ máxima foi de $0,4 \mathrm{~mol} \mathrm{~m}^{-2} \mathrm{~s}^{-1}$ e $\mathrm{g}_{\mathrm{m}}$ foi de $0,47 \mathrm{~mol} \mathrm{~m}^{-2} \mathrm{~s}^{-1}$.

Assim sendo, pode-se estabelecer, para genótipos de feijão comum cultivados no Brasil, de Phaseolus vulgaris var. mexicanus, em condições bem hidratadas, sob saturação luminosa, temperaturas do ar em torno de $25{ }^{\circ} \mathrm{C}$ e bem supridas em nutrientes, que os valores acima citados e apresentados na tabela 1, para as variáveis fotossintéticas $\mathrm{F}_{\mathrm{v}} / \mathrm{F}_{\mathrm{m}}, \mathrm{A}_{\max }$, $\mathrm{g}_{\mathrm{s}}$ e $\mathrm{g}_{\mathrm{m}}$, são semelhantes aos de Phaseolus vulgaris var. aborigineus apresentados na literatura, apesar do centro de origem, base genética e características morfo-fisiológicas distintas nos dois grupos (Norman et al. 1995).

\section{Literatura citada}

Bernacchi, C., Singsaas, E.L., Pimentel, C., Portis Jr, A.R. \& Long, S. 2001. Improved temperature response functions for models of rubisco-limited phtosynthesis. Plant, Cell and Environment 24: 253-259.
Farquhar, G.D., von Caemmerer, S. \& Berry, J.A. 1980. A biochemical model of photosynthetic $\mathrm{CO}_{2}$ assimilation in leaves of $\mathrm{C}_{3}$ species. Planta 149: 78-90.

Flexas, J., Ribas-Carbó, M., Diaz-Espejo, A., Galmés, J. \& Medrano, H. 2008. Mesophyll conductance to $\mathrm{CO}_{2}$ : current knowledge and future prospects. Plant, Cell and Environment 31: 602-621.

Genty, B., Briantais, J.M. \& Baker, N.R. 1989. The relationship between the quantum yield of photosynthetic electron transport and quenching of chlorophyll fluorescence. Biochemical Biophysical Acta 990: 87-92.

Long, S.P. \& Bernacchi, C.J. 2003. Gas exchange measurements, what can they tell us about the underlying limitations to photosynthesis? Procedures and sources of errors. Journal of Experimental Botany 54:2393-2401.

Lynch, J., Gonzaléz, A., Tohme, J.M. \& Garcia, J.A. 1992. Variation in characters related to leaf photosynthesis in wild bean populations. Crop Science 32: 633-640.

McCree, K.J. 1986. Measuring the whole-plant daily carbon balance. Photosynthetica 20: 82-93.

Maxwell, K. \& Johnson, G.N. 2000. Chlorophyll fluorescence - a pratical guide. Journal of Experimental Botany 51: 659-668.

Norman, M.J.T., Pearson, C.J. \& Searle, P.G.E. 1995. The ecology of tropical food crops. Cambridge University Press, Cambridge.

O'Toole, J.C., Ozbun, J.L. \& Wallace, D.H. 1977. Photosynthetic response to water stress in Phaseolus vulgaris. Physiologia Plantarum 40: 111-114.

Pastenes, C. \& Horton, P. 1996. Effect of High Temperature on Photosynthesis in Beans. 1. Oxygen Evolution and Chlorophyll Fluorescence. Plant Physiology 112: 1245-1251.

Pham Thi, A.T., Pimentel, C. \& Silva, V. 1982. Effects of Water Stress on Photosynthesis and Photorespiration of Atriplex nummularia, a $\mathrm{C}_{4}$ plant. Photosynthetica 16:334-342.

Pimentel, C., Laffray, D. \& Louguet, P. 1999. Intrinsic water use efficiency at the pollination stage as a parameter for drought tolerance selection in Phaseolus vulgaris. Physiologia Plantarum 106: 184-189.

Pimentel, C., Ribeiro, R.V., Santos, M.G., Oliveira, R.F. \& Machado, E.C. 2004. Effects of changes in the photosynthetic photon flux density on net gas exchange of Citrus limon and Nicotiana tabacum. Brazilian Journal of Plant Physiology 16: 77-82.

Pimentel, C., Bernacchi, C. \& Long, S. 2007. Limitations to photosynthesis at different temperatures in leaves of Citrus lemon. Brazilian Journal of Plant Physiology 19: 141-147. 
Rohacek, K. 2002. Chlorophyll fluorescence parameters: the definitions, photosynthetic meaning, and mutual relationships. Photosynthetica 40: 13-29.

Sage, R.F., Sharkey, T.M. \& Seemann, J.R. 1988. The invivo response of Ribulose-1,5-Bisphosphate carboxylase activation state and the pool sizes of photosynthetic metabolites to elevated $\mathrm{CO}_{2}$ in Phaseolus vulgaris L. Planta 174: 407-416.

Santos, M.G., Ribeiro, R.V., Machado, E.C. \& Pimentel, C. 2009. Photosynthetic parameters and leaf water potential of five common bean genotypes under mild water deficit. Biologia Plantarum 53: 229-236.

Schreiber, U., Bilger, W. \& Neubauer, C. 1994. Chlorophyll fluorescence as a non-intrusive indicator for rapid assessment of in vivo photosynthesis. In: E.D. Schulze \& M.M. Caldwell (eds.). Ecophysiology of photosynthesis. Springer-Verlag, Berlin, pp. 49-70.
Vassey, T.L. \& Sharkey, T.D. 1989. Mild water stress of Phaseolus vulgaris plants leads to reduced starch synthesis and extractable sucrose phosphate synthase activity. Plant Physiology 89: 1066-1070.

Vieira, C. 1998. Adubação mineral e calagem. In: C. Vieira, T.J. Paula Júnior \& A. Borém (eds.). Feijão: aspectos gerais e cultura no estado de minas. Editora UFV, Viçosa, pp. 123-152.

Wentworth, M., Murchie, E.H., Gray, J.E., Villegas, D., Pastenes, C., Pinto, M. \& Horton, P. 2006. Differential adaptation of two varieties of common bean to abiotic stress: II. Acclimation of photosynthesis. Journal of Experimental Botany 57: 699-709.

Wullschleger, S.D. 1993. Biochemical limitations to carbon assimilation in $\mathrm{C}_{3}$ plants - a retrospective analysis of the $\mathrm{A} / \mathrm{C}_{\mathrm{i}}$ curves from 109 species. Journal of Experimental Botany 44: 907-920. 